\title{
Estimation of Structural Dynamics of a Model Boom of Dragline DRE-23 by Output-Only System Identification Methods.
}

\author{
Zakir Faruquee \\ Department of Mechanical, Automotive \\ and Materials Engineering \\ University of Windsor \\ Windsor, N9B 3P4 \\ Ontario, Canada \\ E-mail:faruqu2@uwindsor.ca
}

\author{
Professor Hal Gurgenci \\ Department of Mechanical Engineering \\ CRC Mining \\ The University of Queensland \\ St. Lucia QLD 4072 \\ Australia \\ E-mail: h.gurgenci@uq.edu.au
}

\begin{abstract}
Two output -only system identification methods namely Canonical Variate Analysis (CVA) and Frequency Domain Decomposition (FDD) were used to estimate the dynamics (Mode shape, natural frequency and damping ratio) of the model boom of the dragline DRE 23. The boom was excited separately with an impulse hammer and with an electrodynamic shaker with chirp, random and simulated field excitations. In all cases, the excitations as well as the responses of the model boom were measured. The dynamics were obtained from the response measurements using Output-Only methods as well as from both the excitations and responses using conventional modal analysis methods. In all cases, the estimations of the dynamics by Output-Only methods were comparable if not better than those estimates obtained by the convention modal analysis methods.
\end{abstract}

\section{Introduction.}

The dynamic characteristics (natural frequency, mode shape and damping ratio) are important parameters of design. They can also be used to monitor the safe operation and reliable maintenance of machines and structures. The dynamic characteristics (resonance frequency, damping ratio and mode shape) of a mechanical system are usually estimated by fitting a model to the Frequency Response Function, a function derived from the input excitation (excitations) and output response (responses) of the system under investigation. The conventional modal analysis requires the measurement of the response (responses) as well as the excitation force (forces). The methods can be applied to a structure that can be easily excited into vibrations. The force is measured instantaneously by a load cell during its application to the structure. The response of the excitation is measured by an accelerometer (accelerometers) and from the inputoutput data, the dynamic characteristics of the system are obtained.

However, some mechanical structures like dragline machines, offshore structures, bridges and nuclear power plants are very difficult to excite and the forces acting on them during their normal operations are not possible to measure. Nevertheless, the response of these structures can be measured quite comfortably. Several algorithms have been developed [1,2,3 ] which estimates the dynamic characteristics of the system by analyzing the response data only and have been applied to limited cases. These techniques are known as the Output-Only methods in the mechanical engineering discipline. In this study, two of these methods have been used to characterize the model boom of the dragline machine DRE -23 for the first time. The output - only methods used in this study are discussed briefly in the following section.

\section{Mathematical Model}

The Canonical Variate Analysis(CVA) and the Frequency Domain Decomposition (FDD) Output-Only methods were used in this study.

\subsection{Canonical Variate Analysis (CVA)}

The stochastic discrete time state space model can be written, when the input $u(k)$ is not known as follows [1].

$$
\begin{aligned}
& \left\{x_{k+1}\right\}=[A]\left\{x_{k}\right\}+\left\{w_{k}\right\} \\
& \left\{y_{k}\right\}=[C]\left\{x_{k}\right\}+\left\{v_{k}\right\}
\end{aligned}
$$

where 
$\left\{x_{k}\right\}$ represents the state vector of dimension $\mathrm{n}$

$\left\{w_{k}\right\}$ zero-mean process noise

$\left\{v_{k}\right\}$ zero-mean measurement noise

$[A]$ and $[C]$ are the state space and the output

matrices, respectively. In addition to the state-space matrices, there are two special matrices that play an important role in Output-Only identification. These are observability and controllability matrices. In general, a state at a sample time $\mathrm{t}=\mathrm{p} \Delta \mathrm{t}$ is observable if and only if the following observability matrix,

$\left[O_{p}\right] \in \square^{m p \times n}$, has the rank of $\mathrm{n}$ :

$$
\left[O_{p}\right]=\left[\begin{array}{l}
{[C]} \\
{[C][A]} \\
{[C][A]^{2}} \\
\cdot \\
{[C][A]^{P-1}}
\end{array}\right]
$$

The state-space system controllability in general is expressed in terms of a controllability matrix, which is built up from the matrices A and B in Equation (1). For an Output-Only system, the inputs are neither measurable nor controllable. Nevertheless, in analogy with regular state-space formulation, we define a controllability matrix for output-only systems as

$$
\left[C_{p}\right]=\left[\begin{array}{llll}
{[G] \quad[A][G] \quad \ldots .} & \left.[A]^{P-1}[G]\right]
\end{array}\right.
$$

where

$$
\begin{aligned}
& C_{p} \in \square^{n \times p r} \quad \text { Controllability Matrix } \\
& {[G]=E\left[\left\{x_{k+1}\right\}\left\{y_{k}\right\}^{T}\right]} \\
& E[.]=\text { Expected value operator }
\end{aligned}
$$

The matrices $\left[O_{p}\right]$ and $\left[C_{P}\right]$ are assumed to be of rank $\mathrm{n}$. Here, the goal is to determine the matrices $[A]$ and $[C]$ from the output measurement responses only. The model order $\mathrm{p}$ are determined by plotting the assumed model number against the damping ratio. The minimum model order corresponding to which damping ratio becomes constant is selected [2,9]. This problem is also known as the stochastic realization problem. The eigenvalues and the observed parts of the eigenvectors of the matrix $[A]$ completely characterize the dynamics of the system. The eigenvalue decomposition of $[A]$ is given by

$$
[A]=[\Phi][\Lambda][\Phi]^{-1}
$$

The discrete eigenvalues $\lambda_{k}$ on the diagonal of $[\Lambda]$ can be transformed into continuous eigenvalues or system poles $\mu_{k}$ by the following equation

$$
\lambda_{k}=e^{\mu_{k} \Delta t}
$$

Taking natural logarithm of both sides

$$
\begin{aligned}
& \ln \left(\lambda_{k}\right)=\mu_{k} \Delta t \\
& \mu_{k}=\sigma_{k}+j \omega_{k}=\frac{\ln \left(\lambda_{k}\right)}{\Delta t}
\end{aligned}
$$

where $\sigma_{k}$ is the damping factor of the $\mathrm{k}$-th mode $\omega_{k}$ is the damped natural frequency of the k-th mode. The damping ratio of the $\mathrm{k}$-th mode is given by the equation

$$
\xi_{k}=-\frac{\sigma_{k}}{\sqrt{\omega_{k}^{2}+\sigma_{k}^{2}}}
$$

The mode shape $\{\psi\}$, of the r-th mode at the sensor locations are the observed parts of the system eigenvectors $\{\phi\}$ of $[\Phi]$. The Henkel matrix is created from the measured output data using the correlation matrix of the measured output vector $[1,4]$ :

$$
\left[R_{k}\right]=\frac{1}{M} \sum_{t=0}^{M-k-1}\left\{y_{t+k}\right\}\left\{y_{t}\right\}^{T}
$$

The Henkel matrix is then written as 


$$
\left[H_{p p}\right]=\left[\begin{array}{lll}
{\left[R_{1}\right]} & {\left[R_{2}\right]} & {\left[R_{p}\right]} \\
{\left[R_{2}\right]} & {\left[R_{3}\right]} & \\
{\left[R_{p}\right]} & {\left[R_{p+1}\right]} & {\left[R_{2 p-1}\right]}
\end{array}\right]
$$

and its singular value decomposition is expressed as

$$
\left[\begin{array}{ll}
H_{p p}
\end{array}\right]=\left[\begin{array}{ll}
{\left[U_{1}\right]} & {\left[U_{2}\right]}
\end{array}\right]\left[\begin{array}{ll}
{\left[S_{1}\right]} & {[0]} \\
{[0]} & {\left[S_{2}\right]}
\end{array}\right]\left[\begin{array}{l}
V_{1}^{T} \\
V_{2}^{T}
\end{array}\right]
$$

where

$$
\begin{aligned}
& S_{1}=\operatorname{diag}\left(\sigma_{1} \cdots \ldots \sigma_{n}\right) \quad \text { and } \sigma_{1} \geq \sigma_{2} \geq \cdots \sigma_{n} \geq 0 \\
& S_{2}=\operatorname{diag}\left(\sigma_{n+1} \cdots \sigma_{p N_{\text {resp }}}\right) \quad \text { with } \quad \sigma_{n+1} \geq \sigma_{n}
\end{aligned}
$$

Then, the CVA method start with the SVD of the normalised Hankel matrix [1,3,4].

$$
\left[\bar{H}_{p p}\right]=\left[W_{1}\right]\left[H_{p p}\right]\left[W_{2}\right]^{T}=\left[[ \overline { U } _ { 1 } ] [ \begin{array} { l } 
{ \overline { U } _ { 2 } ] }
\end{array} ] \left[\begin{array}{l}
{\left[\bar{S}_{1}\right]} \\
{[0]}
\end{array}\right.\right.
$$

where $\quad\left[W_{1}\right]$ and $\left[W_{2}\right]$ are two user-defined invertible weighting matrices of size pxm.

These weighting matrices can be determined from the following is the Toeplitz matrices, for which, the entries constant along each diagonal can be determined from equation.

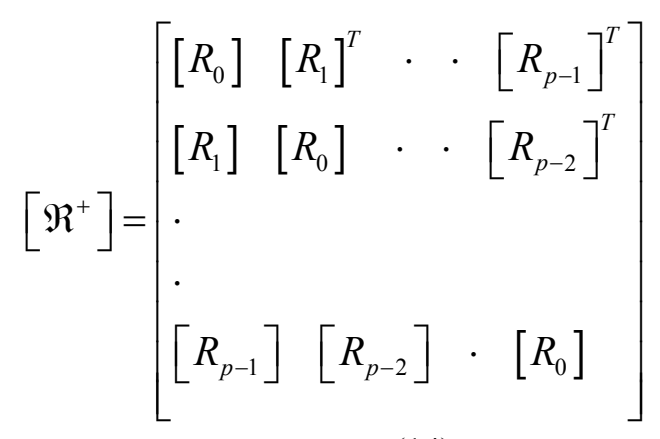

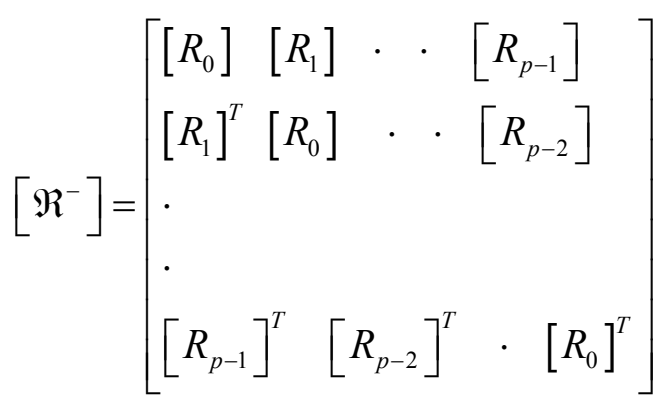

Assuming the full-rank factorisation of

$$
\begin{aligned}
& {\left[\mathfrak{R}^{+}\right] \text {and }\left[\mathfrak{R}^{-}\right]} \\
& {\left[\mathfrak{R}^{+}\right]=\left[L^{+}\right]\left[L^{+}\right]^{T}} \\
& {\left[\mathfrak{R}^{-}\right]=\left[L^{-}\right]\left[L^{-}\right]^{T}}
\end{aligned}
$$

The CVA weighting is then as follows

$$
\begin{aligned}
& {\left[W_{1}\right]=\left[L^{+}\right]^{-1}} \\
& {\left[W_{2}\right]=\left[L^{-}\right]^{-1}}
\end{aligned}
$$

An estimate of the observability matrix is then given

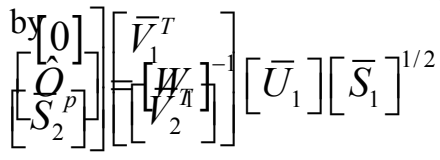

The system matrices are then estimated up to similarity transformation, using the shift structure of $\left[\hat{O}_{p}\right]$. Then the system matrices can be calculated as below.

$[C]=$ first block row of $\left[\hat{O}_{p}\right]$

The $[A]$ is obtained by the following equation $\left[\hat{O}_{p-1}^{\uparrow}\right]=\left[\hat{O}_{p-1}\right][A]$

where, $\left[\hat{O}_{p-1}\right]$ is obtained by deleting the last row of $\left[\hat{O}_{p}\right]$ and $\left[O_{p-1}^{\uparrow}\right]$ is the upper shifted matrix obtained by deleting the first row.

\subsection{Frequency Domain Decomposition}


The relationship between the unknown inputs $x(t)$ and the measured response $y(t)$ can be expressed as follows [6],

$$
\left[G_{y y}(j \omega)\right]=[H(j \omega)]\left[G_{x x}(j \omega)\right][H(j \omega)]^{H}
$$

$G_{x x}(j \omega) \in \square^{r \times r}$ is the Power Spectral

Density (PSD) of the input, and $r$ is the number of inputs. For Output-Only methods the values of $r$ are not required. It is introduced here only to derive the FDD method.

$$
\left[G_{y y}(j \omega)\right] \in \square^{m \times m} \text { is the PSD matrix of }
$$

the $\mathrm{m}$ responses.

$[H(j \omega)] \in \square^{m \times r}$ is the FRF matrix and $\mathrm{H}^{\mathrm{H}}$ denotes the complex conjugate pair.

The FRF can be written in pole/residue form [8]

$$
H(j \omega)=\sum_{k=1}^{n} \frac{R_{k}}{j \omega-\lambda_{k}}+\frac{\bar{R}_{k}}{j \omega-\bar{\lambda}_{k}}
$$

where $\mathrm{n}$ is the number of modes, $\lambda_{k}$ is the pole and $R_{k}$ is the residue.

$$
R_{k}=\phi_{k} \gamma_{k}^{T}
$$

where

$\phi_{k} \square$ mode shape vector

$$
\gamma_{k} \square \text { Modal participation vector }
$$

If the input is assumed as white noise then its PSD becomes a constant matrix, i.e., $G_{x x}(j \omega)=\mathrm{C}$, and equation becomes [5]:

$$
\left[G_{y y}(j \omega)\right]=\sum_{k=1}^{n} \sum_{s=1}^{n}\left[\frac{R_{K}}{j \omega-\lambda_{k}}+\frac{\bar{R}_{K}}{j \omega-\bar{\lambda}_{k}}\right] C\left[\frac{R_{s}}{j \omega-\lambda_{s}}\right.
$$

Using the Heaviside partial fraction theorem, equation can be re-written as follows

$$
\left[G_{y y}(j \omega)\right]=\sum_{k=1}^{n} \frac{A_{k}}{j \omega-\lambda_{s}}+\frac{\bar{A}_{k}}{j \omega-\bar{\lambda}_{k}}+\frac{B_{k} \quad \begin{array}{c}
\text { spaced modes are geometrically orthogonal. If these } \\
\text { assumptisns were not satisfied, the PSD }
\end{array}}{-j \omega-\lambda_{k} \text { approxposination into SDOF system would only be an }}
$$

where $A_{K}$ is the k-th residue matrix of the output PSD. The residue matrix is an $m \times m$ hermitian and is given by

$$
A_{k}=R_{k} C\left(\sum_{s=1}^{n} \frac{\bar{R}_{s}^{T}}{-\lambda_{k}-\bar{\lambda}_{s}}+\frac{R_{s}^{T}}{-\lambda_{k}-\bar{\lambda}_{s}}\right)
$$

The contribution from the $\mathrm{k}$-th mode is given by

$$
A_{k}=\frac{R_{k} C \bar{R}_{k}^{T}}{2 \alpha_{k}}
$$

where; $\alpha_{k}$ is minus the real part of the pole $\lambda_{k}=-\alpha_{k}+j \omega_{k}$. In the case of light damping, the residue becomes proportional to the mode shape vector:

$$
A_{k} \propto R_{k} C \bar{R}_{k}=\phi_{K} \gamma_{k}^{T} C \gamma_{K} \phi_{k}^{T}=d_{k} \phi_{K} \phi_{k}^{T}
$$

where; $d_{k}$ is a scalar constant. At certain frequency $\omega_{k}$, usually a limited number of modes typically one or two contribute significantly. Assuming the set of modes that contributes significantly by $\operatorname{sub}(\omega)$, the response spectral density can be written as

$$
G_{y y}(\omega)=\sum_{k \in \operatorname{sub}(\omega)} \frac{d_{k} \phi_{k} \phi_{k}^{T}}{j \omega-\lambda_{k}}+\frac{\bar{d}_{k} \bar{\phi}_{k} \bar{\phi}_{k}^{T}}{j \omega-\bar{\lambda}_{k}}
$$

After estimating the power spectral density matrix from the output responses, the next step is to decompose the PSD matrix by Singular Value Decop̄ppositjon (SVD) technique. The spectral density matrix is decomposed into a set of auto spectral degree of freedom (SDOF) system. This is true in the case where the loading is by white noise, the structure is lightly damped and the mode shapes of the closely 


$$
\hat{G}_{y y}(j \omega)=U_{i} S_{i} U_{i}^{H}
$$

where the matrix $U_{i}=\left[u_{i 1}, u_{i 2}, \cdots u_{i m}\right]$ is a unitary matrix holding the singular vectors for the SDOF systems. For example, $U_{i j}$ is holding the singular values for the auto spectral density function corresponding to the $\mathrm{j}^{\text {th }}$ mode. Near resonances, either the resonance mode alone or with a close mode will dominate the response. If only the $\mathrm{k}$-th mode is dominating, then there will be only one term in equation. Thus in this case the first singular vector $U_{i 1}$ is an estimate of the mode shape. $\hat{\phi}=u_{i 1}$

and the corresponding singular value is obtained from the auto power spectral density function of the corresponding single degree of freedom system.

\section{Physical Model}

The model of the boom used in this study is shown in Figure1 which is $1 / 25$ th of the actual boom. The boom was $6 \mathrm{~m}$ long consisted of hollow square tubes. The end of the boom was pinned to a rigid Y-frame, and was supported by cables at nodes at 110 and 210 as shown in Figure 2.
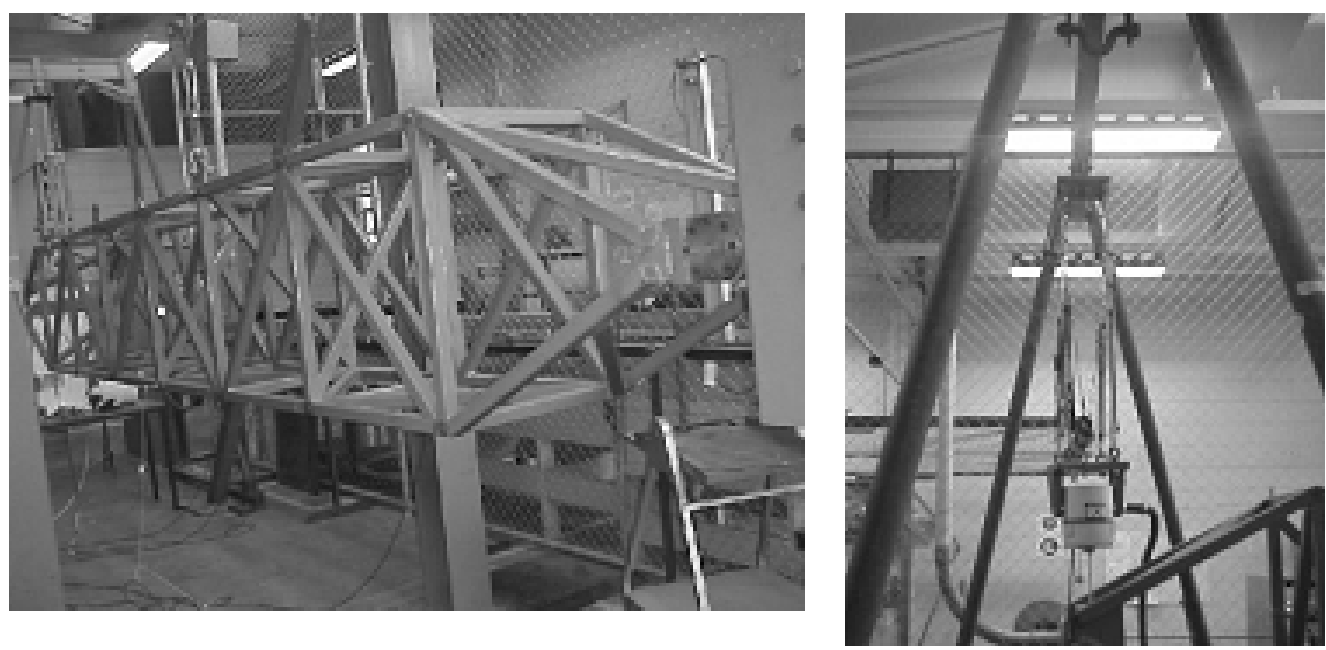

Figure 1: The experimental boom with the shaker attached

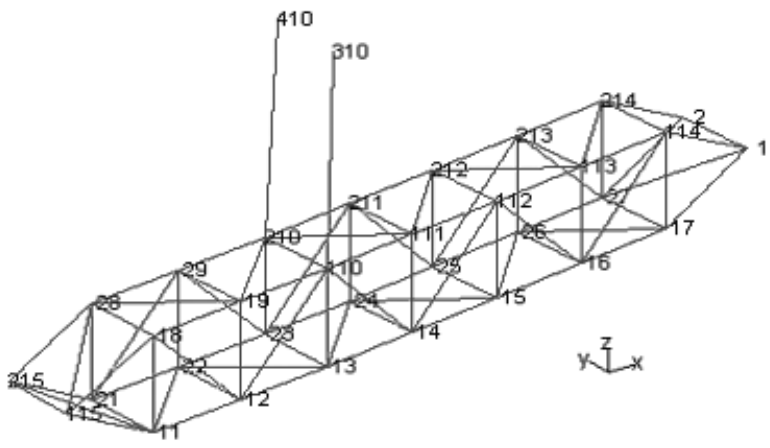

Figure 2: Wire frame of the boom 


\section{Results and Discussions}

This study was conducted by exciting the boom with different kind of excitations. For impact hammer testing, the boom was excited at one nodal point and responses were measured at 9 nodal points by 9 accelerometers. The impact hammer excitation and a typical response of the boom are shown in Figures $3 \mathrm{a}$ and $3 \mathrm{~b}$. The estimation

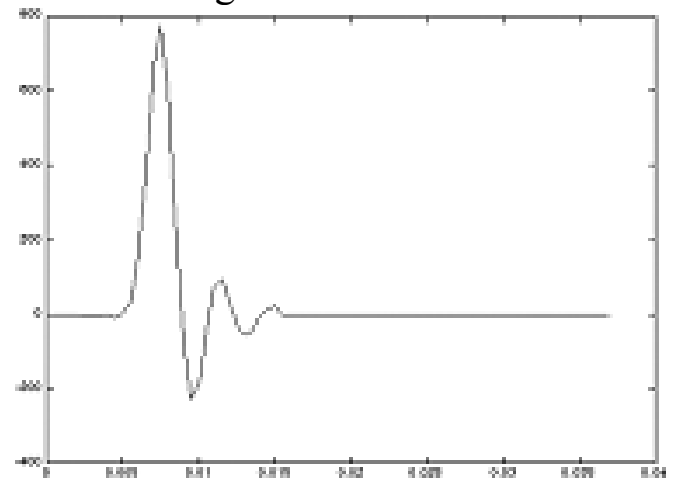

Figure 3a. Impact hammer excitation of natural frequencies, damping ratios and mode shapes obtained by FDD and CVA methods from the responses due to the chirp, random and ambient excitations of the model boom are given in Table 1. A detailed description of the measurements and excitations can be found in reference [2]. HB, VB, T represents horizontal bending, vertical bending and torsion respectively and $1,2, .$. represents first, $2^{\text {nd }}$ etc.

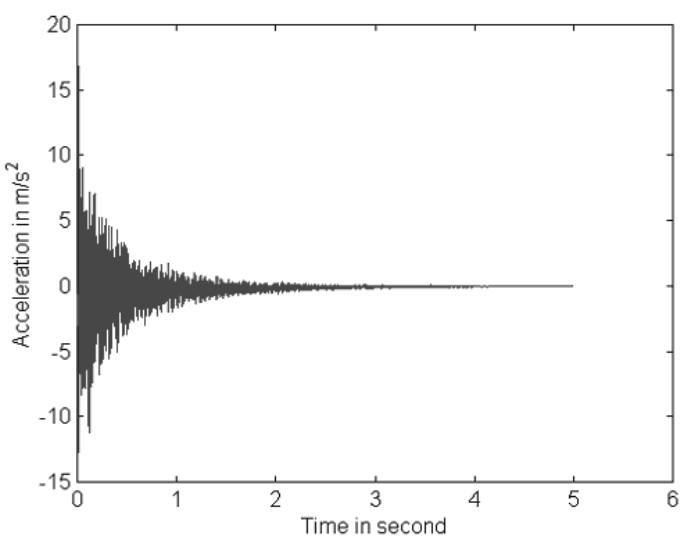

Figure 3b. Response of boom

Table 1:Combined Results of Output-Only Identification tests carried out in this study

\begin{tabular}{|c|c|c|c|c|c|c|c|c|c|c|c|c|c|}
\hline & & HB1 & $V B 1$ & $T 1$ & HB2 & $V B 2$ & $V B 3$ & HB3 & $V B 4$ & $V B 5$ & $C B$ & $T 2$ & HB4 \\
\hline \multirow[t]{2}{*}{ Chirp } & $F D D$ & 4.69 & 14.1 & 40.6 & 53.1 & & 80.5 & & 87.5 & & 103 & 118 & 123 \\
\hline & $C V A$ & 4.59 & 13.6 & 40.5 & 53.1 & & 80.1 & & 87.4 & & 103 & 118 & 123 \\
\hline \multirow[t]{2}{*}{ Random } & $F D D$ & & 14.8 & 40.6 & 54.7 & 61.7 & 80.5 & & 88.5 & & 103 & 118 & \\
\hline & $C V A$ & & 14.1 & 40.9 & 55.0 & 61.9 & 81.8 & & 87.5 & & 103 & 118 & \\
\hline \multirow[t]{2}{*}{ Ambient } & $F D D$ & 1 & 14.7 & 42.0 & 53.7 & 61.5 & 80.1 & 87.9 & 88.9 & 97.9 & 103 & 118 & 124 \\
\hline & $C V A$ & 4.34 & 14.8 & 42.0 & 53.8 & 62.8 & 80.6 & 87.9 & 88.3 & 98.9 & 103 & 118 & 124 \\
\hline \multicolumn{2}{|c|}{ Average Value } & 4.38 & 14.3 & 41.1 & 53.9 & 62.0 & 80.6 & 87.9 & 88 & 98.4 & 103 & 118 & 124 \\
\hline
\end{tabular}

Table 1(continued)

$\begin{array}{lllll}H B 5 & V B 6 & H B 6 & T 3 & T 4\end{array}$

\begin{tabular}{lllllll} 
Chirp & $F D D$ & 128 & 153 & 173 & 181 & 191 \\
& $C V A$ & 128 & 153 & 172 & 182 & 191 \\
Random & $F D D$ & & 153 & & 181 & 191 \\
& $C V A$ & & 153 & & 181 & 191 \\
\multirow{4}{*}{ Ambient } & FDD & & 154 & 173 & 182 & 190 \\
& $C V A$ & & 153 & 173 & 181 & 191 \\
\multirow{5}{*}{ Average Value } & $\mathbf{1 2 8}$ & $\mathbf{1 5 3}$ & $\mathbf{1 7 3}$ & $\mathbf{1 8 1}$ & $\mathbf{1 9 1}$
\end{tabular}


The dynamics of the experimental model boom was obtained by Pole/Residue complex model [2,7]. The Frequency Response Function (FRF) and phage against frequency due to excitation at $28 \_y$ and response at $29 \_\mathrm{y}$ are given in Figure 4. The predicted dynamics by the pole/residue model are compared with the Output - Only methods in Table 2.

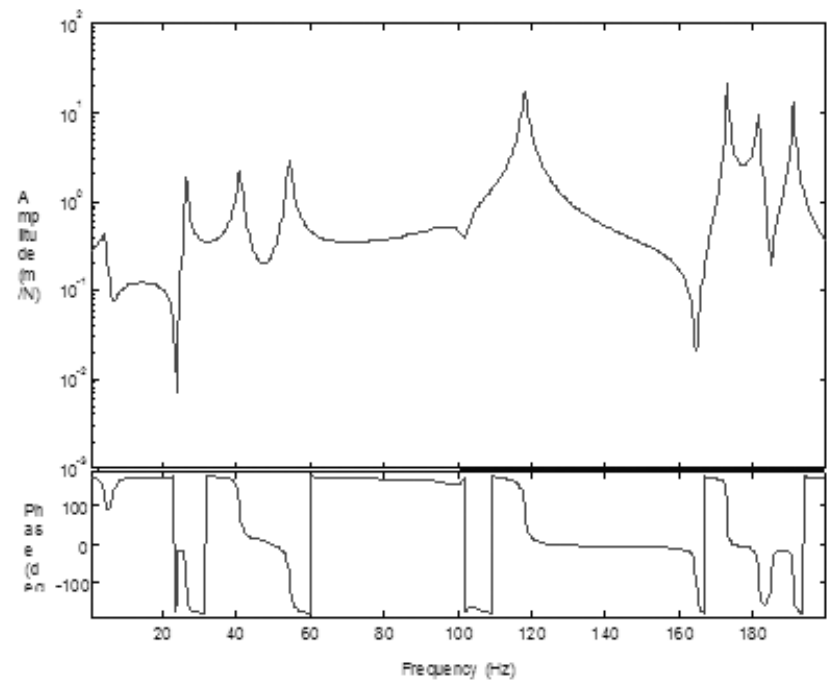

Figure 4: FRF and phase against frequency due to excitation at 28_y and response at 29_y.

Table 2: Comparison between Conventional Modal Analysis and Output-Only Methods

Pole-residue Output-Only

\section{HB1 $V B B 1 \quad T 1 \quad H B 2 \quad V B 2$}

\subsection{1}

4.38

$13.540 .5 \quad 54.4 \quad 61.5$

$\mathrm{VB} 3$
80.7

\section{7}

$\begin{array}{lll}80.7 & 87.7 \\ 80.6 & 87.9 & 88\end{array}$

VB5

CB T2

$\begin{array}{lll}103 & 118 & 126\end{array}$

14.341 .153 .962

Table 2: (continued)

$\begin{array}{lllll}H B 5 & V B 6 & H B 6 & T 3 & T 4\end{array}$

Pole-residue

153173181191

Output-Only

$\begin{array}{lllll}128 & 153 & 172 & 182 & 191\end{array}$

\section{Conclusion:}

Series of experiments were conducted to extract the dynamics of the model boom by conventional modal analysis as well as by Output - Only methods. The test data obtained through (a) Random (b) chirp (c) scaled ambient and (d) impact hammer excitations. The primary objectives were to validate the estimations obtained by the Output - Only methods. The important findings were

In all cases, the extraction of dynamics by the output only methods were accurate and comparable with the data obtained by conventional methods
Mode visualization by Output - Only methods approached the actual mode of boom (3-dimensional) as the number of accelerometers were increased.

Estimation of natural frequencies by FEA was higher as it was very difficult to represents the joints of the suspension cable in FEA. However, the first four modes were MAC paired with the conventional as well as the Output - Only methods.

CVA method worked better than the FDD method under noise excitation, however, FDD method was able to estimate closely spaced and repeated mode. 
The overall, performance of the Output - Only methods were found excellent.

\section{Acknowledgement}

The authors express their sincere gratitude to Center for Mining Equipment and Technology (CMTE) for proving the necessary fund to conduct the study, Structural Vibration Solutions for providing the precommercial version of ARTeMIS software and the university of Queensland for other logistic supports.

\section{References}

[1] L. Hermans, H. V. Auweraer, and M. Abdelghani, "Modal Testing and Analysis of a car under operational conditions", Proceedings of the $16^{\text {th }}$ Modal Analysis Conference (IMAC XVI), Santa Barbara, California, February 3-5,1998.

[2] Z. Faruquee, "Implementation of Output-Only Identification Methods with Demonstration on the Experimental boom", MengSC thesis, The University of Queensland, Australia, 2002

[3] P. V. Overscchee, and B. D. Moor, "Subspace Algorithms for Stochastic Identification Problem", Automatica,Vol. 29, No. 3, pp 649-660.

[4] H. Akaike, "Markovian Representation of Stochastic Processes by Canonical Variables", SIAM Journal of Control, Vol. 13, No. 1, pp 162-172, 1975.

[5] R. Brincker, P. Andersen and N. Moller, "Output Only Modal Testing of a Car Body subjected to Engine Excitaton" Proceedings of the $18^{\text {th }}$ International Modal Analysis Conference (IMAC XVIII), USA, 2000.

[6]J. S. Bendat, and A. J. Piersol, "Random Data Analysis and Measurement Procedure", John Willy and Sons, Inc., USA, 2000.

[7] E. Blames, "New Results on the Identification of Normal Modes from Experimental Complex Modes", Mechanical Systems and Signal Processing, 11(2), pp 229 - 243, 1997.

[8]N. M. M. Maia, and J. M. M. Silva, "Theoratical and Experimental Modal Analysis", Research Studies Press Limited, England, 1997.

[9] Manual of pre-commercial version of ARTeMIS, Structural Vibration Solutions, 1999. 